\title{
PRIMARY ORBITAL LYMPHOMA - A CHALLENGING DIAGNOSIS
}

\author{
St. Vylkanov', K. Trifonova², K. Slaveykov², D. Dzhelebov² \\ 'Department of Neurosurgery, Trakia University - Stara Zagora, Bulgaria \\ ${ }^{2}$ Department of Ophthalmology, Trakia University - Stara Zagora, Bulgaria \\ ${ }^{3}$ First Department of Internal Diseases and General Medicine, Trakia University - Stara Zagora, Bulgaria
}

\begin{abstract}
Background and purpose: The occurrence of primary orbital lymphoma comprises approximately $1 \%$ of non-Hodgkin's lymphoma and $8 \%$ of extranodal lymphoma. The vast majority of orbital lymphomas are of B-cell origin, of which extranodal marginal zone B-cell lymphoma is the most common subtype. The purpose of this paper was to present the diagnostic challenges in a case of orbital lymphoma. Case presentation: An 84year -old woman with orbital tumour was operated on after a long period of inappropriate treatment. It was later diagnosed as B-cell lymphoma. Conclusion: Orbital lymphoma can be easily mistaken for another ocular disease due to the slowly progressing non-specific complaints of the patients. We should be alert to the possibility of this ocular diagnosis when we are presented with an elderly patient with proptosis.
\end{abstract}

Key words: B-cell lymphoma, challenge, non-specific, elderly

Corresponding author: K. Slaveykov, First Department of Internal Diseases and General Medicine, 11 Armeyska Street, 6000 Stara Zagora, Bulgaria, e-mail: kirilslaveykov@gmail.com

\section{BACKGROUND}

$\mathrm{O}$ rbital lymphoma is reported as the most common malignant tumor of the ocular adnexa, constituting $55 \%$ of all orbital tumors [14]. The occurrence of primary orbital lymphoma, on the other hand, is exceedingly rare and comprises approximately $1 \%$ of non-Hodgkin's lymphoma and $8 \%$ of extranodal lymphoma [2]. Orbital lymphomas usually present in the age group of $50-70$ years, with a slight female preponderance [9]. They are the most common primary orbital tumor in adults 60 years of age and older [8].

The vast majority of orbital lymphomas are of Bcell origin $(97 \%)$, of which extranodal marginal zone B-cell lymphoma of mucosa-associated lym- phoid tissue type (MALT) (59\%) is the most common subtype, followed by diffuse large B-cell lymphoma $(23 \%)$, follicular lymphoma (9\%), and mantle cell lymphoma (5\%) [18].

Most tumors present as slowly growing, painless, orbital masses and follow a largely indolent course [9]. Progressive proptosis, decreased visual acuity, diplopia, and restricted ocular mobility are the usual presenting features. The majority of tumors are unilateral; however, bilateral involvement has been reported in $5 \%$ of the cases. Ninety percent of the patients present with localized disease. The most frequent tumor location is the superior lateral quadrant, and the superior rectus is the most commonly involved extraocular muscle [20]. The condition may be primary, involving one or both orbits only, or secondary 
if there are one or more identical foci elsewhere in the body; a substantial proportion of apparently primary lesions will develop disease elsewhere within a few years [12].

Lymphoproliferative lesions, whether benign or malignant, usually mold to surrounding orbital structures rather than invade them. Reactive lymphoid hyperplasias and low-grade lymphomas often have a history of slow expansion over a period of months to years. Orbital imaging reveals a characteristic puttylike molding of the tumor to normal structures. Bone erosion or infiltration is usually not seen except with high-grade malignant lymphomas [3].

Low-grade lymphomas such as extranodal marginal zone B-cell lymphoma and follicular lymphoma have a good prognosis, whereas high-grade lymphomas (diffuse large B-cell lymphoma and mantle cell lymphoma) are associated with a poor prognosis. When managing solitary low-grade lymphomas, radiotherapy is the treatment of choice. Chemotherapy, with or without radiotherapy, should be chosen for disseminated and high-grade lymphomas [18].

The purpose of this paper was to present the diagnostic challenges in a case of orbital lymphoma.

An 84-year-old woman was admitted to the Neurosurgical department of the University Hospital in Stara Zagora, Bulgaria in June, 2018. She was given a working diagnosis - tumour of the right orbit. She had been treated for nearly two years before that by several ophthalmologists for "ocular inflammation". The patient complained of progressive reduction of visual acuity of the same eye and slowly enlarging painless mass of the upper lateral part of the orbit and eyelid. She claimed that she had trauma to the same eye and she connects the start of the symptoms with it. She had hyperemic S-shaped eyelid (Fig. 2 a) of the right eye and proptosis of the right eyeball with interomedial displacement. There was a palpable rubbery mass of the upper eyelid which extended into the upper orbital tissue. The patient could not close her eyelids completely and this would lead to hyperemia of the conjunctiva and stinging and burning sensation. There was reduction in the lateral movement of the eyeball. No organomegaly or lymph node enlargement was found. The rest of the cranial nerves and neurological assessment was normal.

A computer tomography with contrast material was performed (Fig. 1). The mass formation laterally in the orbit and periorbitally absorbed contrast material with increased density. The right lateral muscle and the optic nerve were infiltrated.

Paraclinical test results showed PT - 12,4 sec, INR - 1,09, glucose $-5,92 \mathrm{mmol} / \mathrm{l}$, Creatinine -122
umol/L, Urea - 8,6 mmol/l, ASAT - 51,5 U/l, ALAT - 29,2 U/I, GGT - 993,0 U/l, Total Protein - 65,3 $\mathrm{g} / \mathrm{l}$, Albumin - 33,7 g/l, K+ - 4,4 mmol/l, Na+ - 137

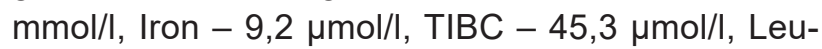
cocytes $-6,73 \times 10^{\wedge} 9 / /$, Lymphocytes - 1,31 $\times 10^{\wedge} 9 / /$, Erytrocytes $-3 \mathrm{~m} 5 \times 10^{\wedge} 12$, Hemoglobin - 107,0 $\mathrm{g} / \mathrm{l}, \mathrm{HCT}-0,33 \mathrm{l} / \mathrm{l}, \mathrm{MCH}-30,6 \mathrm{pg}, \mathrm{MCV}-94,3 \mathrm{fl}$, Platelets $-221,0 \times 10^{\wedge} 9 / \mathrm{l}$.

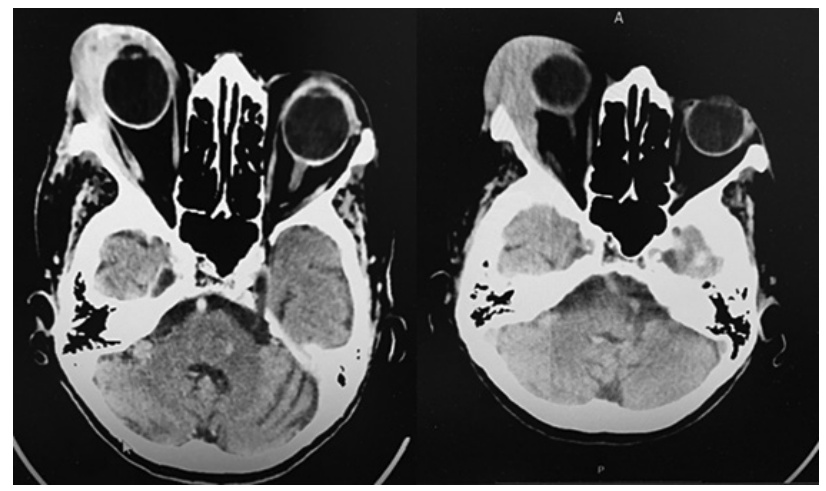

Fig. 1. A CT-scan with contrast material

An operation was performed under general anesthesia. A subbrow S-Shaped incision (Fig. 2 b) was made to obtain access to the tumour mass involving the superolateral part of the orbit. A tumor formation with rigid-elastic consistency resulting from the soft tissues, with secondary propagation into the orbit was reached. The neurosurgeuns suspected by the clinical presentation of the tumour that it originated from the lacrimal gland. However, the lacrimal gland was intact and the tumour molded around its tissue (Fig. 2 b). An attempt to remove the whole intraorbital part of the tumor was made and partially the involved soft tissues of the eyelid (Fig. 2 c). At the end of the operation an attempt for blepharoraphy was performed in order to relieve the symptomatic complaints of the patient (Fig. 2 d).

Histologic diagnosis was initially performed on $\mathrm{H}$ \& E-stained paraffin sections. The pathomorphological signs corresponded mostly with lymphoid hyperplasia. However, Background:malignant lymphoproliferative disorder was discussed. The material was sent for immunohistochemical analysis in the National Specialized Hospital for Active Treatment of Haematologic diseases in Sofia. Their immunomorphological results showed that the material is indolent extranodal marginal zone B-cell lymphoma. The patient was referred to an oncohematologist for complete clinical staging and treatment. Primary orbital lymphomas are a rare subset of tumors constituting 1-2\% of nonHodgkin's lymphoma. They are mostly indolent Bcell lymphomas presenting with gradual progressive 


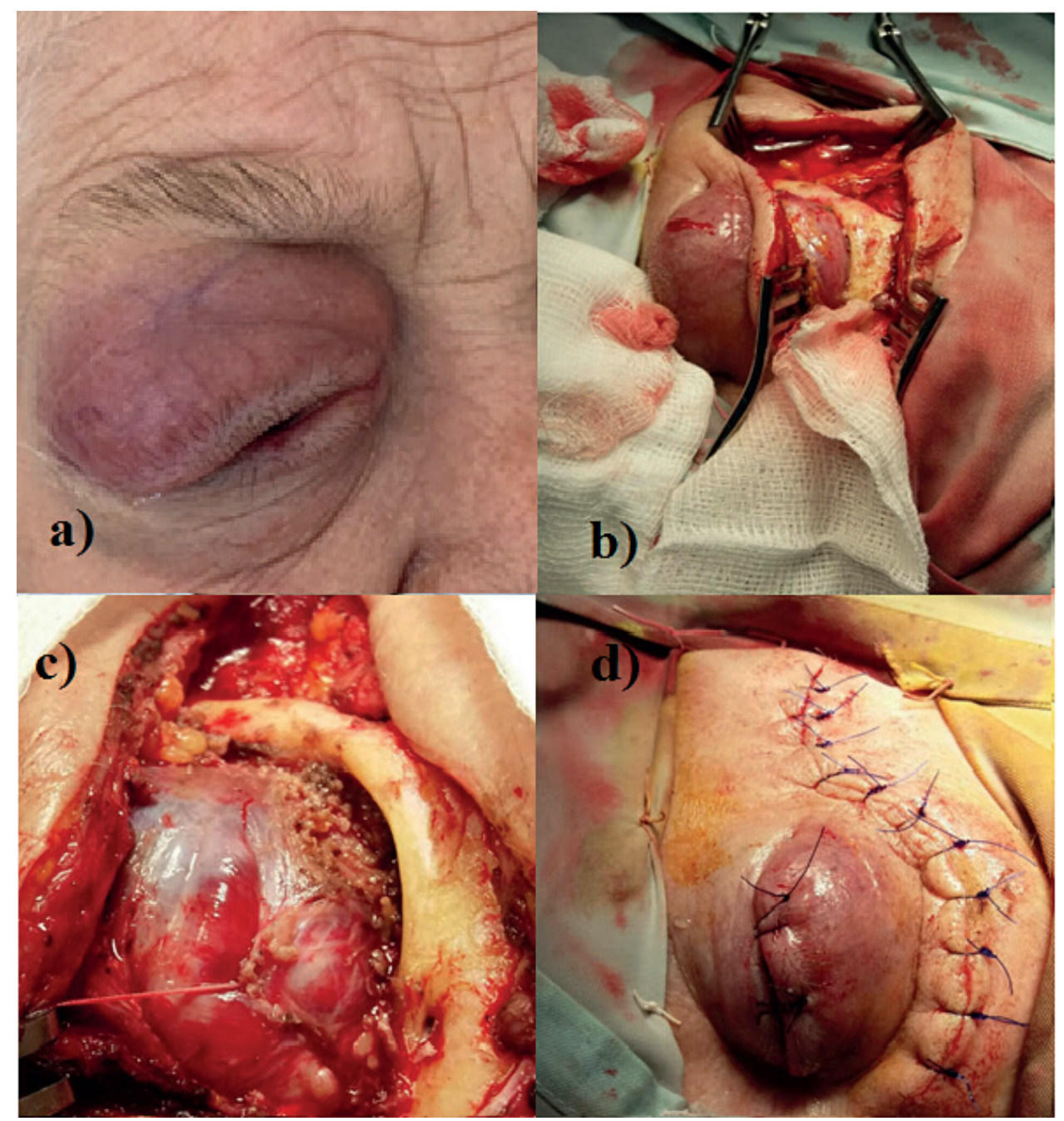

Fig. 2. a) preopative S-shaped eyelid; b) the orbital mass after subbrow S-shaped incision; c) partial removal of the orbital mass; d) sutured incision with attempted blepharoraphy

proptosis, decreased visual acuity, restricted ocular mobility, and diplopia. The role of surgery is mainly for obtaining a biopsy. Most of these tumors require multimodality treatment including chemotherapy, radiation, or both, which have major role.

\section{DISCUSSION}

As you can see by the case we presented, orbital lymphoma sometimes can be a challenging diagnosis as it is a rare presentation of lymphoma. Unspecific symptoms, local infiltration, chronic progression may mimic other more common orbital diseases and often make the diagnosis difficult.

A number of cases have been reported in which these symptoms have been mistakenly interpreted as being secondary to an orbital infection whilst, in fact, other pathology was present $[4,14]$. That is why, proptosis, especially unilateral proptosis, should always lead to a radiological scan in case of malignancy. However, mistakes are still possible [4]. A Cohort study of 26 cases diagnosed with orbital or adnexal lymphoma at Meir Medical Center between 1993 and 2007 showed that in five of these cases lymphoma was misdiagnosed on neuroimaging. According to the authors epiphora or chemosis in the presence of an orbital mass should alert the ophthalmologist to suspect lymphoma [6].

Idiopathic orbital inflammation is a benign non-infective inflammatory condition, which often manifests borderline morphological characteristics [13]. Dacryoadenitis and diffuse inflammation subtypes are easily misdiagnosed as lymphoma because of similar clinical and imaging findings [1]. Before the advent of immunophenotyping and molecular diagnostic techniques, MALT lymphomas were frequently (mis)diagnosed as reactive lymphoid hyperplasia or "pseudotumors" because of their cellular heterogeneity and presence of reactive germinal centers [15]. For all lymphoproliferative lesions, an open biopsy is preferred to allow retrieval of an adequate tissue specimen, which is used to establish a diagnosis and 
to characterize the lesion's morphologic, immunologic, cytogenetic, and molecular properties [3].

Orbital lymphoma mimicking lacrimal gland tumor have also been described based on clinical and radiological features $[19,21]$ The accepted clinicoradiological criteria used for the diagnosis of lacrimal gland fossa lesions might have a certain falsepositive rate, even in recent years [21]. Fine-needle aspiration biopsy or intraoperative biopsy and frozen section diagnosis may help reduce unnecessary lacrimal gland excision [19].

Orbital lymphoma can masquerade as thyroid ophthalmopathy as well. Several such cases have been reported $[5,7,10,23]$. There might be even muscle infitration on computer tomography which suggests Grave's disease as a diagnosis [23]. Even though, it is a rare presentation orbital lymphoma can be in both orbits which makes the differential diagnosis with Grave's ophthalmopathy even harder [7]. Even when elements clearly indicate the presence of thyroid-related ophthalmopathy, disease deterioration should raise a suspicion and always lead to imaging procedures to exclude malignancy.

Surprisingly, orbital lymphoma has been reportedly misdiagnosed as orbital fat pads. There is a need for maintaining a high index of suspicion for orbital lymphoma if tissue excised during a cosmetic blepharoplasty appears abnormal or recurs, especially in patients over 60 years old [11, 17, 22].

\section{CONCLUSION}

Orbital lymphoma can be easily mistaken for another ocular disease due to the slowly progressing nonspecific complaints of the patients. If unspecific orbital symptoms are present, adequate imaging studies followed by early surgical biopsy will contribute to the early diagnosis. We should always be suspicious of this diagnosis especially in patients over sixty years of age with slowly growing mass in the orbit or proptosis non-reacting to specific treatment.

Disclosure summary: The authors have nothing to disclose.

\section{REFERENCES:}

1. Akansel G, Hendrix L, Erickson BA et al. MRI patterns in orbital malignant lymphoma and atypical lymphocytic infiltrates. Eur J Radiol. 2005;53(2):175-181.

2. Ahmed S, Shahid RK, Sison CP et al. Orbital lymphomas: a clinicopathologic study of a rare disease. Am J Med Sci. 2006 Feb; 331(2):79-83.
3. American Academy of Ophthalmology, Basic and Clinical Science Course, 2016.

4. Barkhuysen R, Merkx MA, Weijs WL et al. Plasmablastic lymphoma mimicking orbital cellulitis. Oral Maxillofac Surg. 2008 Sep;12(3):125-8.

5. Boyce PJ. Orbital lymphoma masquerading as thyroid ophthalmopathy. J Am Optom Assoc., 1998, Oct;69(10):666-73.

6. Briscoe D, Safieh C, Ton Y et al, Characteristics of orbital lymphoma: a clinicopathological study of 26 cases. Int Ophthalmol. 2018 Feb;38(1):271-277.

7. Buescu A., P. Teixeira, S. Coelho et al. Orbital lymphoma misdiagnosed as graves' ophthalmopathy, Endocrine Practice, 2001, 7(2):110-2.

8. Demirci H, Shields $\mathrm{CL}$, Shields JA et al. Orbital tumors in the older adult population. Ophthalmology. 2002 Feb; 109(2):243-8.

9. Eckardt AM, Lemound J, Rana M, Gellrich NC. Orbital lymphoma: diagnostic approach and treatment outcome. World journal of surgical oncology. 2013 Dec;11(1):73.

10. Hajduković Z, Kuzmić-Janković S, Kljaković-Avramović $T$ et al. Orbital lymphoma associated with Graves' disease: A case report, Vojnosanit Pregl. 2014 May;71(5):510-4.

11. Hwang CS, Diaz-Marchan P, Marx DP. Mucosa-associated lymphoid tissue lymphoma masquerading as herniated orbital fat. Ophthalmic Plast Reconstr Surg. 2014 MarApr;30(2):e45-7.

12. Kanski J, B Bowling, Kanski's Clinical Ophthalmology A systematic approach, Eight Edition, Elselvier, 2016.

13. Li EY, Yuen HK, Cheuk W. Lymphoproliferative Disease of the orbit. Asia Pac J Ophthalmol (Phila) 2015;4(2):106-111.

14. Mak ST, Wong AC, Tse RK., Diffuse large B-cell lymphoma masquerading as orbital cellulitis. Hong Kong Med J. 2010 Dec;16(6):484-6.

15. Mannami T, Yoshino T, Oshima K et al. Clinical, histopathological, and immunogenetic analysis of ocular adnexal lymphoproliferative disorders: characterization of malt lymphoma and reactive lymphoid hyperplasia. Modern Pathology. 2001 Jul;14(7):641-9.

16. Margo CE, Mulla ZD. Malignant tumors of the orbit. Analysis of the Florida Cancer Registry., Ophthalmology. 1998 Jan; 105(1):185-90.

17. Marival T, Carpentier S, Vandaele S, de Fontaine S. Blepharoplasty revealing orbital lymphoma, Ann Plast Surg. 2013 Mar;70(3):261-3.

18. Olsen TG, Heegaard S. Orbital lymphoma. Surv Ophthalmol. 2019 Jan - Feb;64(1):45-66.

19. Prabhakaran VC, Cannon PS, McNab A et al. Lesions mimicking lacrimal gland pleomorphic adenoma, $\mathrm{Br} \mathrm{J}$ Ophthalmol. 2010 Nov;94(11):1509-12.

20. Priego G, Majos C, Climent F, Muntane A. Orbital lymphoma: imaging features and differential diagnosis. Insights into imaging. 2012 Aug;3(4):337-44.

21. Strianese D, Elefante A, Matarazzo F et al. Orbital Lymphoma Mimicking Lacrimal Gland Pleomorphic AdenomaCase Rep Ophthalmol. 2013 Sep-Dec; 4(3): 109-113.

22. Tong L, Qian J, Adam R. Bilateral orbital lymphoma presenting as recurrence of orbital fat pad after blepharoplasty. Can J Ophthalmol. 2017 Feb;52(1):e9-e11.

23. Wolyniak B, Lewczuk A, Obolonczyk L et al. Orbital lymphoma mimicking Graves' ophthalmopathy: a case report, Central European Journal of Medicine, 2011, 6:826

Accepted: 15 February 2020 